While family medicine has significant experience with PBRNs, it has not found funding support to build the significant research infrastructure required to perform large scale clinical trials that link a large number of PBRNs. Historically, large RCTs have recruited subjects divorced from the clinical care setting and community, and have been costly. The ADFM proposal to support an integrated national research network will enable large scale comparative effectiveness research and patient outcomes research in a cost effective manner. We believe that it has the potential to become a "reusable rocket" to power important future research.

Paul James, MD and the ADFM Research Development Committee, Wilson Pace, MD (DARTNET)

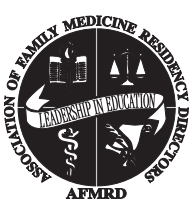

From the Association of Family Medicine Residency Directors

Ann Fam Med 2013;11:283-284. doi:10.1370/afm.1530.

\section{ACCOUNTABLE CARE ORGANIZATIONS: AN OPPORTUNITY FOR SYNERGY}

With the reelection of President Obama, full enactment of the Patient Protection and Affordable Care Act is all but certain. Part of that legislation is the establishment of Accountable Care Organizations (ACOs). These large networks require a minimum of 5,000 Medicare patients, and will assume the total costs for their care in many cases. Over 140 ACOs are already established with over 130,000 physicians and 2.2 million beneficiaries. ${ }^{1}$ Much of the broad legislation governing ACOs has yet to be converted to specific regulations, which will vary between states. Because so much of this change has yet to be decided, and large systems will have to rapidly adapt, ACOs may become a sudden order of business for family medicine program directors.

ACOs are a model of shared risk for costs and savings of a defined population. The more patients a network has, the more easily they can spread the costs of expensive care of the relatively few. In the ACO model, hospitals will shift from revenue centers to cost centers. ACOs have the potential to shift systems to embrace wellness rather than reactive illness care. In the future, we may get reports on how much our patients cost the system, rather than how much revenue we generated with our level-4 visits, inpatient billing, and procedures. Many systems will need to increase their primary care workforce as more patients have health coverage, and systems shift their emphasis to outpatient and preventive care.

Insurance and payment reforms are the first 2 steps in health care reform. The third phase is delivery system reform. ${ }^{1}$ A need to educate those making ACO formation decisions will exist, regarding the value of family medicine residency programs to ACO networks. One of the fundamental objectives of the National Institute of Program Director Development (NIPDD) training is to understand the worth of your program; in the world of ACOs, our 2 principal strengths to promote will be cost-effective care and workforce generation.

To truly provide population health care (rather than just those who come to see us) will require a fundamental shift in perspective for our systems, and most of us as well. Many of us will need to learn new skills; we will need to strongly advocate for resources such as case managers, chronic disease registries, and searchable electronic health records to provide high quality, cost-effective health care to a population. We also need to advocate for payment reforms that truly reflect our value to our systems. If we are not involved in the early formation and leadership of ACOs, we risk maintaining the status quo of huge payment disparities between procedure-based specialists and diverse primary care practices. These disparities discourage future medical students from entering primary care, which eventually will hurt all of our patients.

Residency education about cost-effective care is optimized if the system can provide each resident with clinical quality and cost data on their own panel of patients, rather than having them subsumed under the faculty patient panel. We need to advocate for not just teaching about quality improvement, but doing it in our residency practices

Family medicine has a long and celebrated history of advocating for our patients. With the formation of ACOs, there is a moral imperative that we advocate for systems that reflect our values as a specialty. In the next few months, many decisions will be made that will affect both process values (the rules that govern decision-making processes such as transparency, accountability, and participation) and content values (clinical effectiveness, cost-effectiveness, justice/equality, and autonomy). ${ }^{2}$ We all need to ensure that family medicine has a voice at the table, and that we are proud of the end result. After all, the ACOs created in the next few months will be the ones our graduates will be practicing in for the foreseeable future.

Stepben Scbultz, MD

Stoney Abercrombie, MD, Brian Crownover, $M D_{\text {; }}$

Grant Hoekzema, MD, Nathan Krug, MD, Lisa Maxwell, MD, Michael Mazzone, MD, Karen Mitchell, MD, Todd Shaffer, MD, MBA, Michael Tuggy, MD 


\section{References}

1. Song $Z$, Lee TH. The era of delivery system reform begins. JAMA. 2013;309(1):35-36.

2. Keren R, Littlejohns P. Consideration of social values in the establishment of accountable care organizations in the USA. J Health Organ Manag. 2012;26(3):384-389.

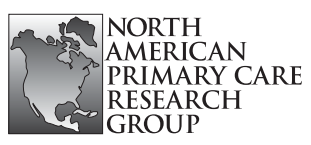

From the North American Primary Care Research Group

Ann Fam Med 2013;11:284-285. doi:10.1370/afm.1537.

\section{PBRNS ENGAGING THE COMMUNITY TO INTEGRATE PRIMARY CARE AND PUBLIC HEALTH}

The 2013 NAPCRG/AHRQ PBRN conference will be held June 18-19, 2013 in Bethesda, Maryland. It promises to provide opportunities to share strategies, methods and results, and learn more about funding opportunities.

Key takeaways from last year's conference were:

Practice-based research networks (PBRNs) are becoming a crucial link between primary care and public health. It has become apparent over the past decade that primary care and public health must work together much more closely than in the past if we are to truly tackle the serious health issues in our communities. The new Institute of Medicine Report, Primary Care and Public Health: Exploring Integration to Improve Population Health, provides a basic roadmap for collaborations between public health agencies, communitybased organizations, and primary care practices. ${ }^{1}$ The report, commissioned by the Health Resources and Services Administration and the Centers for Disease Control and Prevention, gives numerous examples of primary care and public health integration. This theme was emphasized at the NAPCRG PBRN meeting where attendees heard examples of practice-based research collaborate with primary care practices to improve patient and community health; attendees left the conference energized to build future partnerships with public health. Health care reform, whether in the form of the Affordable Care Act or local grassroots efforts to build communities of solution, drives the relationship-building efforts between primary care and public health.

Practice-based research networks have evolved over the past 40 years from a small group of curious family doctors to hundreds of rigorous, well-funded research laboratories. PBRNs have the infrastructure and capacity to bring primary care and public health together to improve the health of patients and community members. It is a testament to the diligent work of our providers and research that PBRNs are now in a position to think and work beyond the walls of their clinic. By collaborating with governmental public health agencies and non-governmental community-based organizations, PBRNs have potential to impact both routine clinical practice and the broader community. PBRNs already engage their providers and patients, and are perfectly poised to engage their communities. Thus, PBRNs can serve as the catalyst to integrate primary care and public health.

Three examples of ongoing PBRN and public health collaborations were presented. First, the early work in the Oklahoma PBRN included an advisory committee member from the state health department. Current work includes a robust primary care extension service that places extension agents into communities to directly work with primary care offices and public health programs. Second, the High Plains Research Network in Colorado developed an asthma toolkit that linked improved primary care practice capacity for diagnosis and treatment to community organizations and schools to increase awareness and self-management. Third, the Canadian Partnership Against Cancer provided an example of a large-scale national collaboration between numerous primary care practices, public health agencies, and consumer groups which lead to improved chronic disease prevention and screening. In addition, several posters and research presentations at the PBRN conference offered growing evidence for improved outcomes through primary care/public health collaborations. Finally, a workshop provided tangible steps for beginning a collaboration between a PBRN and public health and community-based organizations.

The emphasis in health care reform on health promotion and disease prevention brings everyone to the table. PBRNs can serve as the laboratory for testing, implementing, and disseminating locally relevant interventions in the primary care practice and the community setting. Interventions, innovations, and quality improvements in disease prevention and health promotion often require alignment of medical care, self-management, the built environment, and a community approach. Practice-based research networks will be a crucial tool for developing successful models that engage local and broader communities and create linkages between primary care and public health. PBRNs should think beyond the walls of their clinical practices and accept the challenge of expanding their capabilities to build new collaborations that benefit population health. 